LBL-33221

UC-410

LSGN-116

\title{
FINAL ANALYSIS OF THE ALS LATTICE MAGNET DATA
}

\author{
RODERICH KELLER \\ ACCELERATOR and FUSION RESEARCH DIVISION \\ Lawrence Berkeley Laboratory \\ University of California \\ Berkeley, CA 94720
}

MAY 1993

Figith

This work was supported by the Director, Office of Energy Research, Office of Basic Energy Sciences, Materials Sciences Division, of the U.S. Department of Energy under Contract No. DE-AC03-76SF00098. 


\title{
Final Analysis of the ALS Lattice Magnet Data*
}

\author{
Roderich Keller \\ Lawrence Berkeley Laboratory \\ University of California, Berkeley, CA 94720 USA
}

\begin{abstract}
A preliminary analysis of the measured magnet data of the multipole magnets to be installed in the Advanced Light Source (ALS) storage ring had been given in the last conference of this series [1]. That analysis was based on measurements of about one-third of the entire magnet complement, and some of its findings had to be revised after obtaining more data. The present paper gives an account of the integrated fundamental field strengths of all installed storage ring lattice magnets. For the multipole magnets, these values are listed as analytical expressions with four or five parameters, that cover the entire exci-tation range and include residual strength. Based on this final analysis, all gradient and quadrupole magnets were qualified for installation, whereas the sextupole magnets had to be individually trimmed to bring their effective fundamental strengths into the desired tolerance band.
\end{abstract}

\section{INTRODUCTION}

The measurements of all lattice magnets for the Advanced Light Source (ALS) [2] storage ring have been completed since the last conference of this series [3]. For the production magnets, the main concern lies in the magnet-to-magnet reproducibility of their fundamental excitations, whereas the higher-order multipole errors are largely determined by the given pole shapes, optimized in earlier investigations of engineering models of each basic magnet type [4].

The quadrupole and sextupole magnets were measured using rotating, integrating coiis. Their excitation curves are approximated by analytical expressions that include two fitting parameters for the saturation effect and, in the case of quadrupoles, another fitting parameter to compensate for residual excitation. These approximations are more refined than the ones published earlier [1].

All measurements have been taken with rising excitation current, after conditioning every magnet to the maximum power supply current and then reducing the excitation current to zero. For the storage ring gradient magnets elaborate Hall probe scans had to be performed to ascertain sufficient accuracy for the measurements of their quadrupole components. These Hall maps were numerically integrated to derive integrated dipole and quadrupole components, effective lengths, and effective edge angles.

The evaluation procedures and results will be discussed in detail for every magnet type. All nominal magnet parameters given below refer to the standard beam energy of $1.5 \mathrm{GeV}$.

*This work was supported by the Director, Office of Energy Research, Office of Basic Energy Sciences, Materials Sciences Division, U.S. Department of Energy, under Contract No. DE-AC03-76SF00098.

\section{DEFINITIONS}

Integrated strengths of multipole magnets, $\mathrm{d}^{\mathrm{n}-1}$ (BL)/dx ${ }^{\mathrm{n}-1}$, are listed in the tables below as transfer functions $T(I)$ of the excitation current $I[A]$, with $B$, local flux density [T]; $L$, effective length [m]; $x$, horizontal co-ordinate [m]; $n=2$ for quadrupoles; and $n=3$ for sextupoles.

The commonly used $\mathbf{k}$-values are computed from these values as:

$$
k_{n}=\frac{1}{B_{1} \rho L} \frac{d^{n-1}(B ~ L)}{d x^{n-1}}
$$

The beam rigidity is always $B_{1} \rho=5.00536[\mathrm{~T} \mathrm{~m}]$.

The tolerance band for the relative errors of integrated magnet strengths was set to $\pm 1 \times 10^{-3}$ for the $1 \sigma$ width of the actual distribution, with an absolute cut-off limit of $\pm 2 \times 10^{\text {- }}$ ${ }^{3}[5]$.

\section{APPROXIMATION FORMULAE}

Magnetic measurements have been carried out on each of the individual lattice magnets after three times cycling the excitation current from zero to the maximum of the power supply and back to zero. Various excitation values were then set by monotonically raising the current from zero to the desired level. This procedure means that in the course of the measurements a transition from the upper to the lower branch of the hysteresis loop has been made, see Figure 1, and this fact is relevant for all quadrupole measurements.

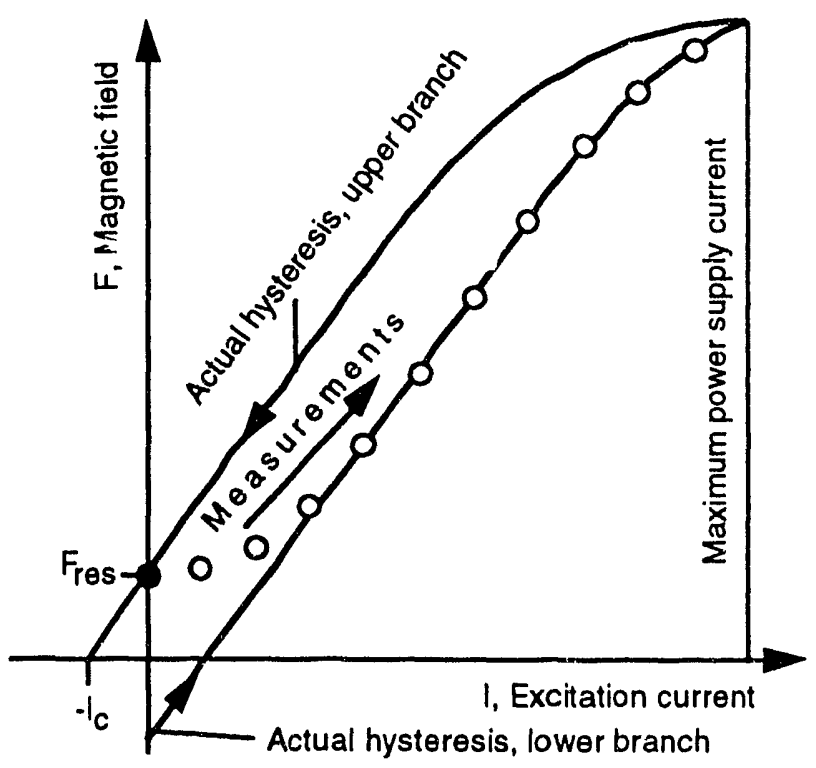

Figure 1. Construction of the lower branch of a hysteresis loop from magnet measurements $F_{i}$, meas (open circles) affected by residual fields. Residual field and saturation $-1-$ 
To reconstruct the lower hysteresis branch for a magnetic quantity $F$ (e.g. integrated gradient for quadrupoles), the data $F_{i}$,meas measured near zero current have to be reduced to ideal data $F_{i}$ by subtracting twice the measured residual value $F_{\text {res. }}$ This reduction then must gradually vanish as the measured points approach the actual hysteresis branch. An exponential expression (2) with a fitting parameter $\mathrm{C}$ was developed to provide the reduction, assuming that ten measurement with running number $i$ have been taken, with beginning saturation effects at the last three values:

$$
F_{i}=F_{i, \text { meas }}-2 F_{\text {res }} / \exp \left\{I_{i} /\left(C I_{c}\right)\right\} \quad[1 \leq i \leq 10]
$$

The coercive current $I_{c}$ (absolute value) is determined by

$$
\mathrm{I}_{\mathrm{c}}=\mathrm{F}_{\mathrm{res}} / \mathrm{T}_{\mathrm{raw}}
$$

with an approximate "raw transfer function" given by:

$$
\mathrm{T}_{\text {raw }}=\left\langle\mathrm{F}_{\mathrm{i} \text {,meas }} / \mathrm{I}_{\mathrm{i}}\right\rangle \quad[4 \leq \mathrm{i} \leq 7]
$$

$C$ is recursively optimized such that the reduced value at the first current, $F_{1}$, matches the average reduced transfer function $T$ of the other values in the linear part of the hysteresis:

$$
\mathrm{F}_{1} /\left(\mathrm{I}_{1}-\mathrm{I}_{\mathrm{c}}\right)=\mathrm{T}_{\text {lin }} \equiv\left\langle\mathrm{F}_{\mathrm{i}} /\left(\mathrm{I}_{\mathrm{i}}-\mathrm{I}_{\mathrm{c}}\right)\right\rangle \quad[4 \leq \mathrm{i} \leq 7]
$$

For the three quadrupole families, the individual optimum $C$ values were separately averaged, and these values were then applied once more to each data set. No residual field measurements had been made with the sextupoles, and therefore the lower excitation values have been given less weight in the evaluation of transfer function coefficients.

To determine a closed expression for the transfer function $T(I)$ at arbitrary exciation currents I over the full range of the constructed hysteresis curve, a saturation term is introduced:

$$
\mathrm{T}(\mathrm{I})=\mathrm{T}_{\text {lin }} /\left\{1+\left(\left(\mathrm{I}-\mathrm{I}_{\mathrm{c}}\right) / \mathrm{I}_{\mathrm{s}}\right)^{\mathrm{A}}\right\}
$$

where $\mathrm{A}$ is a fitting parameter that is kept constant for all magnets of one family ( $A=3$ for quadrupoles) and the "saturation current" $I_{s}$ is individually calculated as: with

$$
\mathrm{I}_{\mathrm{s}}=\left\langle\left(\mathrm{I}_{\mathrm{i}}-\mathrm{I}_{\mathrm{C}}\right) \times\left(\mathrm{T}_{\text {lin }} / \mathrm{T}_{\mathrm{i}}-1\right)^{-1 / \mathrm{A}}\right\rangle \quad[8 \leq \mathrm{i} \leq 10]
$$

$$
\mathrm{T}_{\mathrm{i}}=\mathrm{F}_{\mathrm{i}} /\left(\mathrm{I}_{\mathrm{i}}-\mathrm{I}_{\mathrm{c}}\right)
$$

The optimization of $\mathrm{A}$ is performed by minimizing the differences $R_{i}$ between all individual $F_{i} /\left(I-I_{c}\right)$ values and the determined average transfer function $T\left(I-I_{c}\right)$ from eq. (6):

$$
R_{i}=T\left(I-I_{c}\right)-F_{i} /\left(I-I_{c}\right) \quad[1 \leq \mathrm{i} \leq 10]
$$

The following expression can then be used to compute actual values of the magnet strength of interest for a given power supply current, I:

$$
\mathrm{F}(\mathrm{I})=\mathrm{T}_{\text {lin }} /\left\{1+\left(\left(\mathrm{I} \pm \mathrm{I}_{\mathrm{c}}\right) / \mathrm{I}_{\mathrm{s}}\right)^{\mathrm{A}}\right\} \times\left(\mathrm{I} \pm \mathrm{I}_{\mathrm{c}}\right)
$$

using tabulated values of $T_{\text {lin }}, I_{c}, I_{s}$, and $A$. With Equ. (10) one has to select one of the two branches of the hysteresis curve, depending on operational practice. In the case of ALS, where magnets are set on the upper hysteresis branch after exciting them with maximum current, $I+I_{c}$ is the appropriate choice. For the lower hysteresis branch, $\mathrm{I}-\mathrm{I}_{\mathrm{c}}$ is taken.

Figure 2 shows as an example for one quadrupole, the derived fitting curve $T\left(I-I_{c}\right)$ together with the reduced measured data $F_{i} /\left(I-I_{c}\right)$.

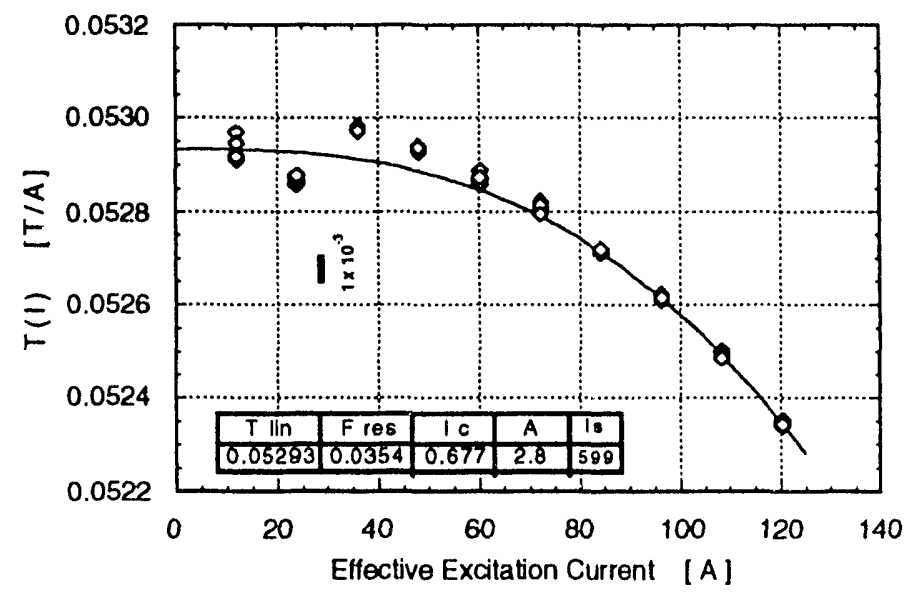

Figure 2. Reduced measured transfer function values and fitting curve for the integrated gradient of quadrupole QF17 as a function of the effective current, $\mathrm{I}-\mathrm{I}_{\mathrm{c}}$.

\section{RESULTS}

\section{Bend Magnets}

The storage ring bend magnet is a straight, parallelended combined-function magnet with the following main parameters: bend angle, $10^{\circ}$; magnetic length, $0.865 \mathrm{~m}$; and effective field edge angles of $2^{\circ}$ (wider toward the outer side). From Hall probe scans [1], [3] at $700 \mathrm{~A}$ excitation current, the main characteristic data, i.e. integrated dipole field, integrated gradient, core field, and core gradient were evaluated. Core is here defined as the central $\pm 250 \mathrm{~mm}$ of the magnet. Field integration was performed numerically, with trapezoidal approximation. Statistics for all produced magnets are given in Table 1. One should note that the measured quantities apply to straight paths along the magnet, whereas the beam follows a curved trajectory. Furthermore, after installation on girders the fringe field of the bend magnets is clipped by adjacent magnets, and this effect changes the actual, integrated quantities by a small but significant amount.

Table 1

Storage Ring Bend Magnets Fundamental Parameters

\begin{tabular}{|c|c|c|c|c|}
\hline & $\begin{array}{c}\text { B L } \\
{[\mathrm{Tm}]}\end{array}$ & $\begin{array}{c}\mathrm{G} \mathrm{L} \\
{[\mathrm{T}]}\end{array}$ & $\begin{array}{c}\mathrm{B} \\
{[\mathrm{T}]}\end{array}$ & $\begin{array}{c}\mathrm{G} \\
{[\mathrm{T} / \mathrm{m}]}\end{array}$ \\
\hline Average & 0.9059 & -3.420 & 1.047 & -4.039 \\
\hline$\sigma_{\text {rel }}\left[10^{-3}\right]$ & 0.58 & 0.60 & 0.45 & 0.57 \\
\hline Max.Rel.Dev. $\left[10^{-3}\right]$ & 1.53 & 1.91 & 1.11 & 1.00 \\
\hline
\end{tabular}

\section{Quadrupoles}

There are three families of quadrupoles with identical cross-sectional shape but different effective lengths: QFA 
(focusing), $0.445 \mathrm{~m}$; QF (focusing), $0.350 \mathrm{~m}$; and QD (defocusing), $0.200 \mathrm{~m}$. The nominal core gradients, $\mathrm{G}=$ $\mathrm{dB} / \mathrm{dx}$, are $15 \mathrm{~T} / \mathrm{m}$ at $400 \mathrm{~A}$ excitation current with $0.621 \mathrm{~T}$ pole tip field (QFA) and $13.4 \mathrm{~T} / \mathrm{m}$ at $89 \mathrm{~A}$ with $0.434 \mathrm{~T}$ pole tip field (QF, QD). The problems with poor magnet-tomagnet reproducibility, reported earlier [1], were solved by using a better suited rotating integral coil with higher quadrupole sensitivity [3]. Statistics for fundamental strengths and averaged parameters of all produced quadrupoles are given in Tables 2 and 3.

Table 2

Storage Ring Quadrupoles Fundamental Statistics

\begin{tabular}{|c|c|c|c|}
\hline & $\begin{array}{c}\text { Average } \\
\mathrm{T}_{\text {lin }} \\
{\left[\mathrm{T} \mathrm{A}^{-1}\right]}\end{array}$ & $\begin{array}{c}\sigma_{\text {rel }} \\
{\left[10^{-3}\right]}\end{array}$ & $\begin{array}{c}\text { Max. Rel. } \\
\text { Deviation } \\
{\left[10^{-3}\right]}\end{array}$ \\
\hline QFA & 0.017223 & 0.49 & 1.1 \\
\hline QF & 0.052922 & 0.61 & 1.2 \\
\hline QD & 0.028754 & 0.68 & 1.3 \\
\hline
\end{tabular}

Table 3

Storage Ring Quadrupoles Average Measured Parameters

\begin{tabular}{|c|c|c|c|c|c|}
\hline & $\begin{array}{c}F_{\text {res }} \\
{[\mathrm{T}]}\end{array}$ & $\begin{array}{c}\mathrm{I}_{\mathrm{c}} \\
{[\mathrm{A}]}\end{array}$ & $\mathrm{C}$ & $\mathrm{A}$ & $\begin{array}{c}\mathrm{I}_{\text {s }} \\
{[\mathrm{A}]}\end{array}$ \\
\hline $\mathrm{QFA}$ & 0.045 & 2.65 & 29.51 & 3.1 & 2250 \\
\hline $\mathrm{QF}$ & 0.035 & 0.661 & 29.58 & 2.8 & 604 \\
\hline $\mathrm{QD}$ & 0.020 & 0.711 & 31.02 & 4.3 & 353 \\
\hline
\end{tabular}

\section{Sextupoles}

All sextupoles belonging to the two families, SF (horizontally focusing) and SD (vertically focusing) are built identically. In addition to their main function, they are equipped with trim windings to produce horizontal and vertical steering and a skew quadrupole excitation, but for this report their sextupole functions only are being discussed. The nominal fundamental parameters for $1.5 \mathrm{GeV}$ beam energy are: $0.484 \mathrm{~T}$ pole tip field $\left(395 \mathrm{~T} / \mathrm{m}^{2}\right)$ at $290 \mathrm{~A}$ excitation with $0.2 \mathrm{~m}$ effective length. After measuring all magnets, the spread of fundamental strengths exceeded the tolerance band by nearly a factor of four. Because the sextupoles are being excited for storage ring operation by two common power supplies it was decided to shunt most of them such as to match the weakest members of each family [6]. Statistics for both cases, without and with shunting, are given in Table 4.

Table 4

Storage Ring Sextupoles Fundamental Statistics

\begin{tabular}{|c|c|c|c|}
\hline & $\begin{array}{c}\text { Average } \\
\mathrm{T}_{\text {lin }} \\
{\left[\mathrm{T} \mathrm{m}^{-1} \mathrm{~A}^{-1}\right]}\end{array}$ & $\begin{array}{c}\sigma_{\text {rel }} \\
{\left[10^{-3}\right]}\end{array}$ & $\begin{array}{c}\text { Max. Rel. } \\
\text { Deviation } \\
{\left[10^{-3}\right]}\end{array}$ \\
\hline SF No Shunt & 0.5361 & 3.7 & 7.1 \\
\hline SF Shunted & 0.5323 & 0.05 & 0.09 \\
\hline SD No Shunt & 0.5362 & 3.3 & 5.0 \\
\hline SD Shunted & 0.5335 & 0.18 & 0.62 \\
\hline
\end{tabular}

Fitting parameters for the transfer functions, separately averaged for the two sextupole families, are given in Table 5. As mentioned above, no actual residual field measurements were made for sextupoles, and the derivation of fitting parameters follows a simpler scheme than outlined in Section III. The effect is insignificant at the operation point of the sextupoles. For the purpose of computing integrated sextupole strengths, $S=d^{2} B / d x^{2}$, from Tables 4 and 5 for a given current, Equation (11) is to be used:

$$
S L=T_{l i n} \times I /\left\{1+\left(I / I_{s}\right)^{A}\right\}+F_{e s}
$$

Table 5

Storage Ring Sextupoles Average Measured Parameters

\begin{tabular}{|c|c|c|c|}
\hline & $\begin{array}{c}\text { F }_{\text {res }} \\
{\left[\mathrm{T} \mathrm{m}^{-1}\right]}\end{array}$ & A & $\begin{array}{c}\mathrm{I}_{\mathbf{S}} \\
{[\mathrm{A}]}\end{array}$ \\
\hline SF & 0.221 & 5 & 797 \\
\hline SD & 0.216 & 5 & 796 \\
\hline
\end{tabular}

\section{CONCLUSION}

Measured magnetic field data of all ALS storage ring lattice magnets were analysed to judge the variance of their fundamental field strengths. A new algorithm was developed that allows reducing the measured magnet strength values to represent one branch (the lower one) only of the hysteresis curve and further to approximate the saturation part of the hysteresis in one closed expression. All lattice magnets for the ALS storage ring could be qualified for installation without observing a particular placement order; the sextupoles, however, had to be shunted to keep their effective fundamental strengths within the $\pm 1 \times 10^{-3}$ tolerance band.

\section{ACKNOWLEDGMENTS}

The author would like to thank J. Tanabe for providing the raw data for this analysis work and R. Alvis for her valuable assistance in data processing and preparing graphs of the results. Thanks are also due to $A$. Jackson and $K$. Halbach for many discussions and advice in defining the evaluation procedures.

\section{REFERENCES}

[1] R. Keller, "Magnetic Data Analysis for the ALS Lattice Magnets" Conference Record of the 1991 IEEE Particle Accelerator Conference, Vol. 4, pp. 2113-2115.

[2] "1-2 GeV Synchrotron Radiation Source, Conceptual Design Report," LBL Pub. 5172 Rev., LBL Berkeley, 1986.

[3] Measurements performed at LBL Berkeley under the guidance of J. Tanabe, D. Nelson, and M.I. Green.

[4] J. Tanabe et al., "Fabrication and Test of Prototype Ring Magnets for the ALS," Conf. Record of the 1989 IEEE Particle Accelerator Conference, Vol. 1, pp. 566-568.

[5] A. Jackson, "Magnetic Field Tolerances in the ALS Storage Ring," ALS LSAP-56, LBL Berkeley, 1989.

[6] J. Tanabe, "Calculation of Shunts," LSME-486A LBL Berkeley (1992). 


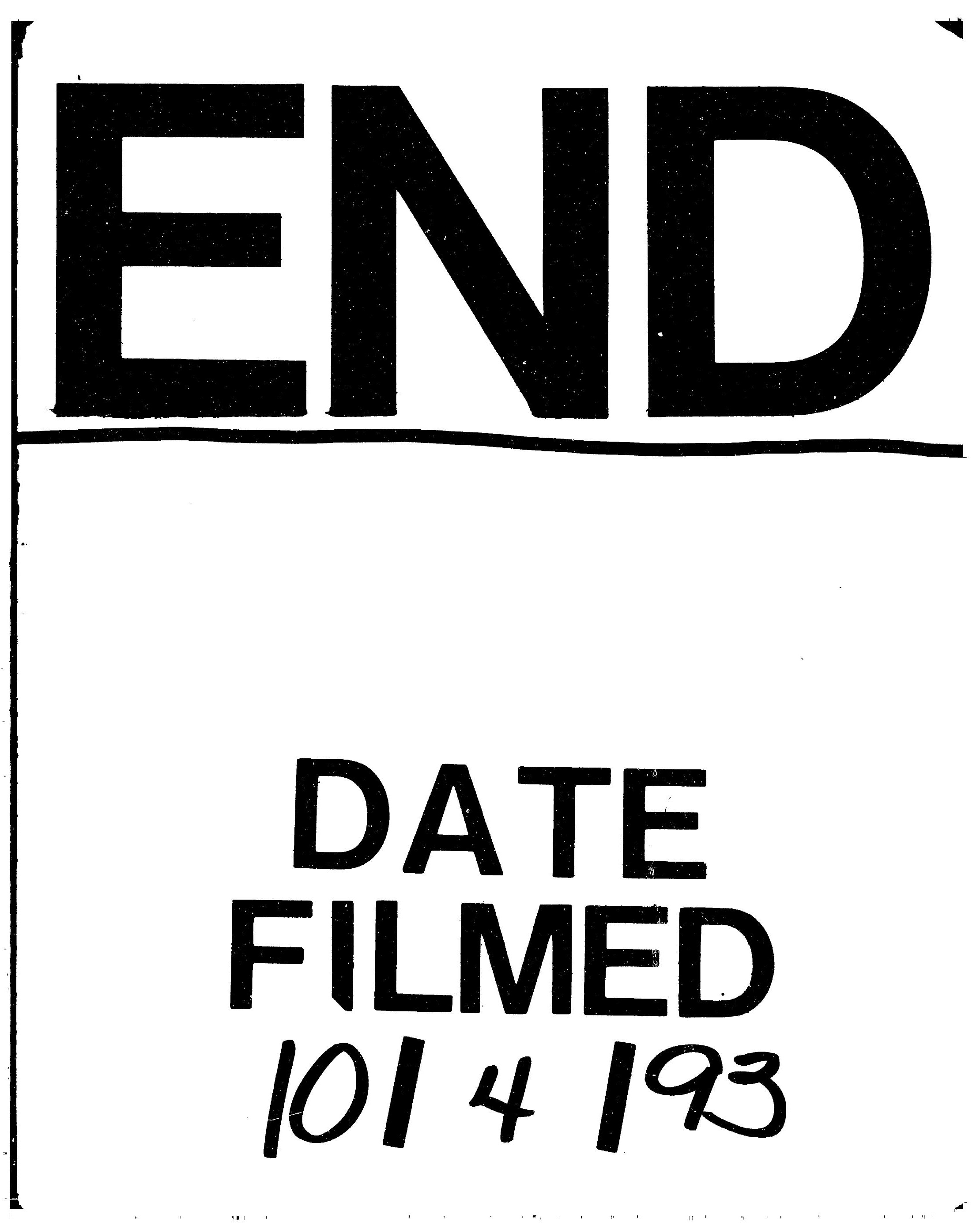


